The Effectiveness of Using Robotics for Career Technology Education in a Middle School STE(A)M Course (Fundamental)

Dr. Jennifer Parham-Mocello, Oregon State University

I am interested in computer science education research related to K-12 curriculum, diversity in computer science, undergraduate computer science learning, and adult and teacher training.

Mr. Ernie Bodle, Oregon State University

I am interested in incorporating more STEM topics in K-12 education to prepare future generations to our ever advancing world. 


\section{The Effectiveness of Using Robotics for Career Technology Education in a Middle School STE(A)M Course (Fundamental)}

Abstract

Using robotics in education allows students to become familiar with multiple topics in science, technology, engineering, and mathematics (STEM). With the use of robotic educational tools in the 8th - 12th grade classrooms, such as Sphero, Anki Cozmo, and Lego Mindstorms, few devices allow students to build the robots' electrical circuits along with constructing and programming. By incorporating electronics into these educational tools, students can learn another fundamental discipline of robotics. For this research, we introduce the Parallax ActivityBot $360^{\circ}$ to the Linus Pauling Middle School career technology education (CTE) course to see if the device promotes STEM. The ActivityBot $360^{\circ}$ incorporates robotics, electronics, computer science, and mathematics into constructing and using the device. Students use the tutorials given online to assemble the robot and to program it with a block-based coding language called BlocklyProp with an option to use the text-based $\mathrm{C}$ language. The main idea is to determine the effectiveness of this tool for CTE courses and how it can change students' interest, enjoyment, confidence, knowledge, and/or motivation to pursue a degree or career in STEM. For this research, we surveyed $8^{\text {th }}$ grade middle school students before and after using the device to see how their opinions and knowledge in STEM changed. From the results, we find that students' confidence in three of the four topics surveyed increased after using the ActivityBot $360^{\circ}$.

Introduction

In the $8^{\text {th }}$ through $12^{\text {th }}$ grade Career Technology Education (CTE) courses, technology can be used to expose students to different topics that could lead to interest in certain STEM careers. Products, such as the Arduino, can be used to expose students to programming and building circuits with various electrical components. The Arduino, and similar devices, can be used to expose students to programing languages, such as C, Scratch, etc. [1]. Block-based programming languages, like Scratch, can also be used as a gateway to introduce students to 
more advanced programming and robotics [1]. Programming and electronics are two of the several topics incorporated into robotics, which gives robotics versatility as a Science, Technology, Engineering, and Mathematics (STEM) learning tool.

The flexibility of robots allows for a variety of uses in education. Some robots can be used as learning assistants, such as Georgia Institute of Technology's intelligent assistant, Jill [2]. Other educational robots can be used for "medical training" or "intelligent toys for preschool children" [2]. This paper focuses on the use of "multi-function suite robots," which are hands-on robots that aim to teach students about robotics by letting those students construct and program their devices [2]. Teaching students with these types of robots can expose them to a variety of STEM topics, such as computer science, electronics, mathematics, etc. [3]. Preconstructed and programmable robots, such as Sphero and the Anki Cozmo, let students apply the robot to solve a variety of problems. Being "one of the most popular" robot to be used in education, Lego Mindstorms allow students to program and construct the robot in a variety of forms [4]. With an abundant amount of literature surrounding the use of Lego Mindstorms, there lacks research on other similar robots in CTE courses. This paper will evaluate the effectiveness of the ActivityBot $360^{\circ}$ in a middle school CTE course.

\section{Motivation for Research}

In 2016, Oregon passed Measure 98 to fund CTE courses in high school and amended the measure in 2017 to include the 8 th grade [5], [6]. CTE courses offer education for a variety of careers, such as health science, business, information technology, STEM, etc. The main goal of CTE is to help students develop skills that can be useful for future careers. CTE can also be used to expose students to concepts that they would be unlikely to see in everyday life, such as electronics, robotics, and/or computer science.

Linus Pauling Middle School, a Corvallis District dual-language (Spanish and English) immersion school, created the Science, Technology, Engineering, Art, and Mathematics (STE(A)M) CTE elective course in response to Measure 98. During the course's second year, a 
robotics intervention was introduced to the course. The ActivityBot $360^{\circ}$ kits were used for this intervention and were provided by Parallax Inc.

The technology company Parallax Inc. wanted research on the effectiveness of the ActivityBot $360^{\circ}$ kit in middle school CTE courses. Parallax wanted to evaluate how engaged students were towards STEM topics and how their engagements would change before and after using the device in the course. They also wanted to find out how students' attitudes towards majoring or working in a STEM related field would change after using the ActivityBot $360^{\circ}$.

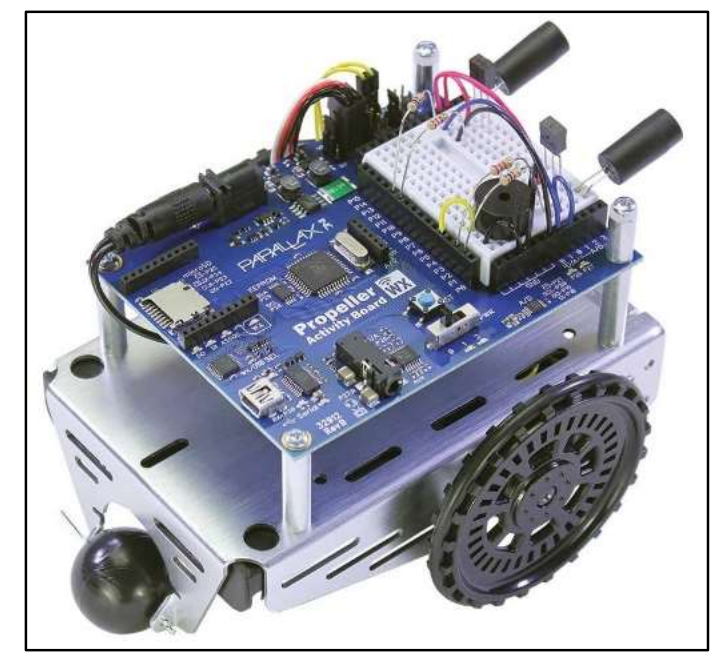

Figure 1. The ActivityBot $360^{\circ}$ with Infrared Sensors and Emitter

Shown in Figure 1, the ActivityBot $360^{\circ}$ allows students to construct the physical robot, construct its circuits, and program the robot. The ActivityBot $360^{\circ}$ kit consists of three wheels, a battery pack, two servo motors, multiple electrical components, a chassis, a Propeller Activity Board WX, and a few tools. The Propeller Board is a programmable printed circuit board (PCB) with a bread board and many ports attached. The ports and bread board allow students to customize and connect a variety of sensors and electrical components, such as the infrared emitter, infrared receiver, buzzer, and resistors to the robot. The parts just listed are shown connected to the device in Figure 1. For writing the programs that control the robot, students can either use the block-based programming language BlocklyProp or use the text-based C language. Block-based languages allow students to program without having to learn syntax that may be too complicated for students who are not yet educated in computer science. The students who have 
prior knowledge in text-based languages can use $\mathrm{C}$ instead of BlocklyProp. Both languages are used in the tutorials provided in the Learn Parallax website. The website also has tutorials on constructing the robot and how to connect each electrical component [7].

Since the ActivityBot $360^{\circ}$ blends electronics, programming, and robotics in one device, students can get a broader understanding of STEM concepts. Few robotic kits expose students to actual electronics, such as electrical components, bread boarding, and assembling circuits, and many robots, like Sphero and the Anki Cozmo, are only programmable. The Lego Mindstorms lets users assemble the robot, but all electronic parts connect with telephone jack like connectors. This makes all the electrical components for the Mindstorms a black box.

\section{Related Works}

Chaudhary et al. [4] evaluate the effectiveness of using the Lego Mindstorms EV3 as a learning tool and a way to get K-8 students interested in STEM. Chaudhary et al. [4] introduced the Lego Mindstorms EV3 to a summer camp for elementary school students. The camp had instructors teach a hands-on curriculum centered around the device for multiple days. Along with surveying the students about their experience, the researchers evaluated students' "computational and logical thinking skills" before and after the summer camp [4]. The evaluations were performed by having students play an online game that focused on computational thinking using Scratch. The research concluded that students' engagement increased after using the EV3 model at the summer camp [4].

In addition to Chaudhary, Zygouris et al. [9] used Lego Mindstorms, but they introduced the Lego Mindstorms NXT to a group of 12-year-old primary school students. The device was used as a hands-on approach in teaching geometry to students. The researchers concluded that students who used the device gained more knowledge in geometry than a control group of students who had normal lecturing without the Lego Mindstorms NXT [9].

In contrast to the research using Lego Mindstorms, Knop et al. [8] had middle school students learn how to build and use a "Neu-pulator platform", which is a robot that resembles a 
human arm. They had the students take a survey before and after the course to see how the students' interests in different topics changed after the intervention, and they implemented daily surveys to measure how general confidence changed throughout the course. Through the surveys, they found "an increase in the level of interest towards robotics among the students, with a significant increase for girls" [8]. They also found that students showed an increase in confidence, "especially in topics related to computer science" [8].

Related research shows that integrating robotics into a classroom can positively influence a student's attitude and knowledge towards STEM and STEM related topics, which is a motivation for using robotics in the pre-college curriculum to teach STEM. The research presented in this paper focuses on the impacts of using robotics to expose electrical engineering and computer science to $8^{\text {th }}$ grade middle school students, and we seek to find similar results as past research when applying the ActivityBot $360^{\circ}$ to a middle school STE(A)M CTE elective course.

Proposed Method \& Experiment

This research evaluates the effectiveness of the ActivityBot $360^{\circ}$ for CTE courses and changing students' attitudes toward STEM. With such a broad research goal to evaluate, we split the overall goal into different sub-goals that are directly related to what the STE(A)M CTE course is trying to achieve. First, we defined effectiveness as a positive change in student interest, enjoyment, confidence, knowledge, and/or motivation to pursue a degree or career in STEM as a result of being exposed to the ActivityBot $360^{\circ}$ and its tutorials. To answer these questions, we asked middle school STE(A)M CTE elective students survey and quiz questions before and after the robotics intervention in the class.

After obtaining IRB approval, a pre-survey, post-survey, and quiz were provided to the students during class time to evaluate the students' attitudes and learning, and all surveys and quizzes were written in both English and Spanish. Surveys were designed to gather demographic data, student opinions, and student attitudes before and after using the ActivityBot $360^{\circ}$. After each survey, students completed a quiz to determine their initial knowledge and how their 
knowledge changed after the robotics intervention in the course. Figure 2 shows an example of a survey question used to measure students' confidence in different fields, and Figure 3 shows an example question from the quiz used to assess their understanding of circuits and the orientation of the LED. Students encountered all the parts and symbols in the quiz, while constructing the ActivityBot $360^{\circ}$ and its circuits. The students took the surveys and quizzes online through Qualtrics.

\begin{tabular}{|l|ccccc|}
\hline \multicolumn{7}{|l|}{ For your grade level, how confident are you in each field? } \\
\hline & $\begin{array}{c}\text { Not Confident } \\
\text { at all }\end{array}$ & $\begin{array}{c}\text { Slightly not } \\
\text { Confident }\end{array}$ & Neutral & $\begin{array}{c}\text { Slightly } \\
\text { Confident }\end{array}$ & Very Confident \\
\hline Robotics & 0 & 0 & 0 & 0 & 0 \\
Electronics & 0 & 0 & 0 & 0 & 0 \\
$\begin{array}{l}\text { Programming / Computer } \\
\text { Science }\end{array}$ & 0 & 0 & 0 & 0 & 0 \\
Mathematics & 0 & 0 & 0 & 0 & 0 \\
\hline
\end{tabular}

Figure 2. Survey Question for Measuring Confidence

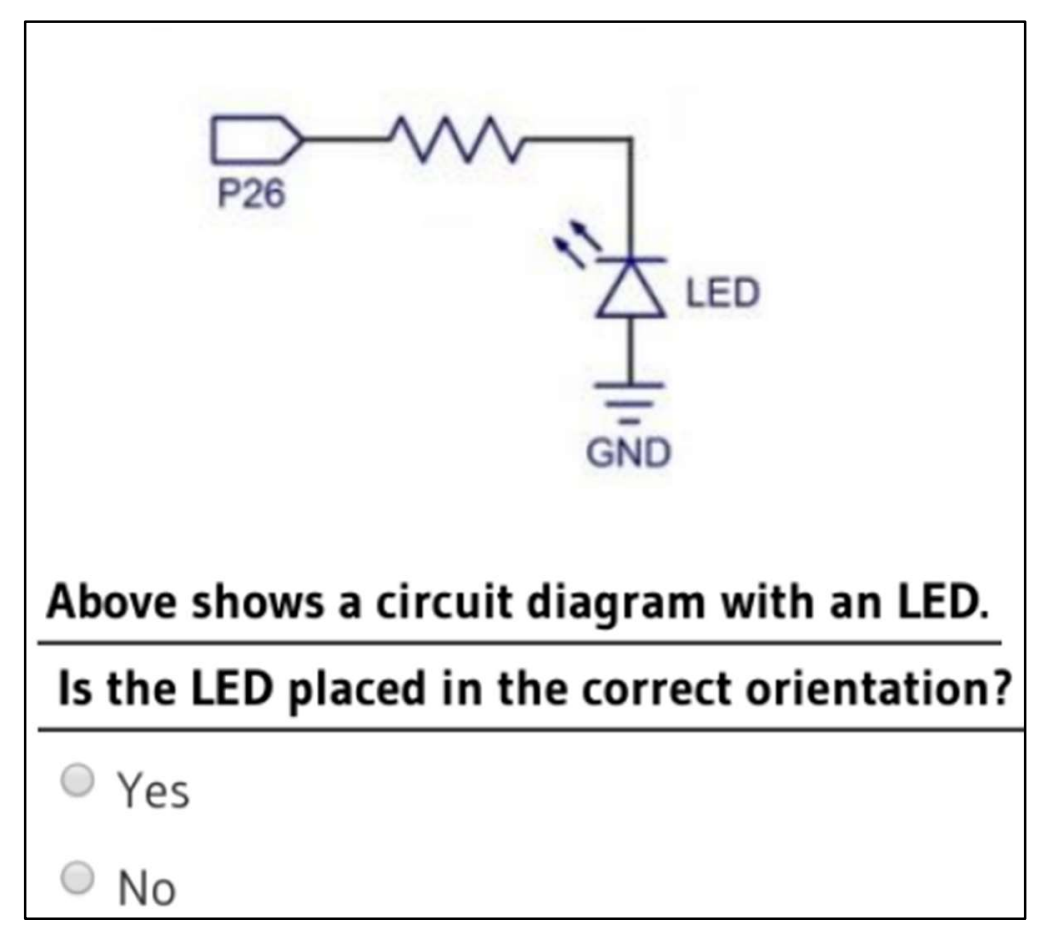

Figure 3. Quiz Question 3 
While surveys and quizzes were reviewed by a licensed teacher and the director of the Oregon State STEM Academy, who runs STEM based summer camps for K-12 students, we recognize that the surveys and quizzes were not tested for reliability and validity using rigorous statistical methods. Before conducting the study, parents received a notification, in both English and Spanish, with an opt-out option for their child's participation in the study.

Before each survey, students were presented with an assent form to fill out through the online survey service, Qualtrics. Assenting students took the pre-survey and the first iteration of the quiz on October 9th, 2018. After taking the pre-survey, students went through the tutorials provided by Parallax with the help of the instructor to construct, program, and set up the circuits to control the ActivityBot $360^{\circ}$. The tutorial shows and explains how to use each part that is provided by the robotics kit. The tutorial teaches students how to use BlocklyProp with an option to use the text-based language $\mathrm{C}$. After the robotics intervention, students took the post-survey and the second iteration of the quiz on January 30th, 2019.

Results and Discussion

Fifty-seven students completed both the pre and post survey and quiz. Out of these 57 students, 15 students were female, and 11 students were Hispanic. There were 20 students who stated that they did not choose to take this elective, and the students who chose to take the elective, chose for various reasons. The majority (33) of the students had not taken a prior STEM based course or camp or did not have prior experience in Electronics. Whereas, there were only 20 students without prior experience in Computer Science and 23 without prior experience in Robotics.

For the 57 students who participated in both the pre and post surveys, we used a Wilcox Signed-Rank Test to determine if there was a change in 1) interest in the STE(A)M elective, 2) enjoyment and confidence in different STEM topics, 3) motivation to pursue different STEM degrees/careers, and 4) electrical and programming knowledge before and after the intervention. This paired test takes two data sets of an identical population and returns a specific p-value. Pvalues that are less than the 0.05 significance level represents a significant change in how 
students answered from pre-survey to post-survey. Since we used the Wilcox Signed-Rank test to answer questions about the students' change in enjoyment, confidence and motivation for multiple STEM degrees and their interest in the elective on the same population, we lowered the p-value to .01 be more conservative in our claim to reject the hypothesis, which we understand increases the chances of a Type II error. While student confidence in mathematics, interest in the STE(A)M elective, and enjoyment and motivation to pursue a degree / career for the topics of robotics, electronics, computer science, and mathematics did not change, there was a change in their confidence in robotics, electronics, and computer science before and after the intervention.

This research finds that students were more confident in robotics, electronics, and computer science after the intervention with returned p-values of 7.413E-05, 6.100E-04, and 1.170E-05 respectively (see Figures 4-6). The confidence levels of 1 to 5 in Figures 4, 5, and 6 correspond to "Not Confident at all" (1) to "Very Confident" (5) respectively. For each of these topics, the average confidence level rose 1 point from "Neutrally Confident" (3) to "Slightly Confident" (4).

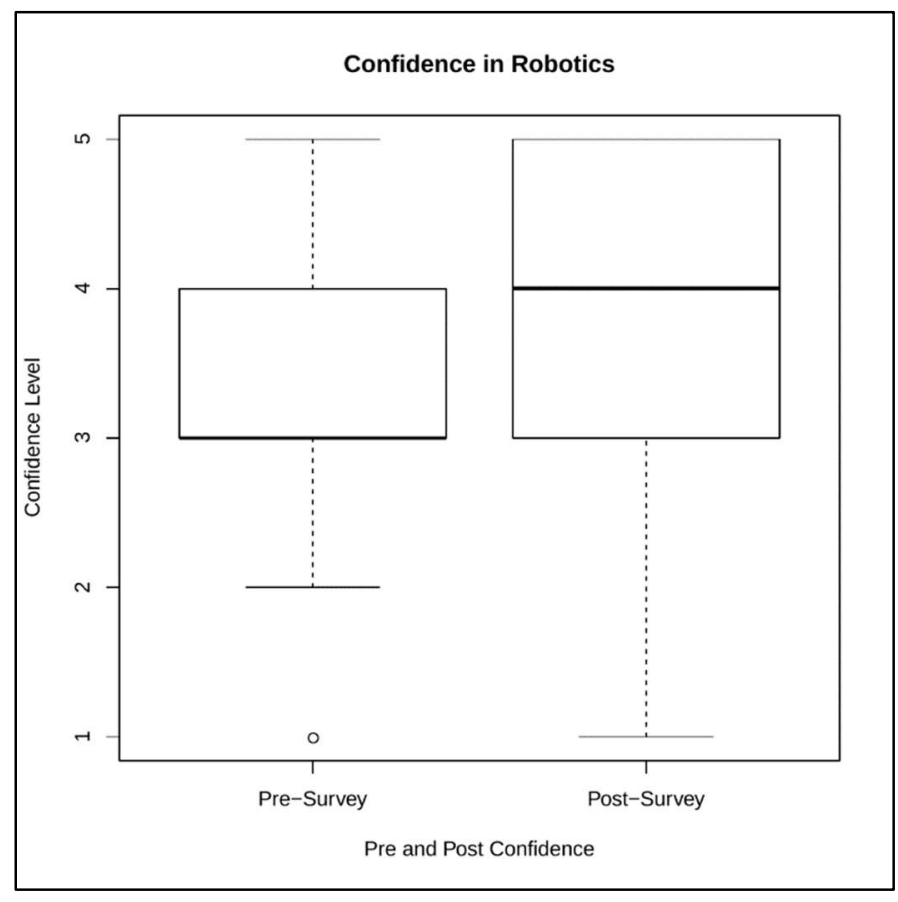

Figure 4. Differences of Pre and Post Survey Results for Confidence in Robotics 


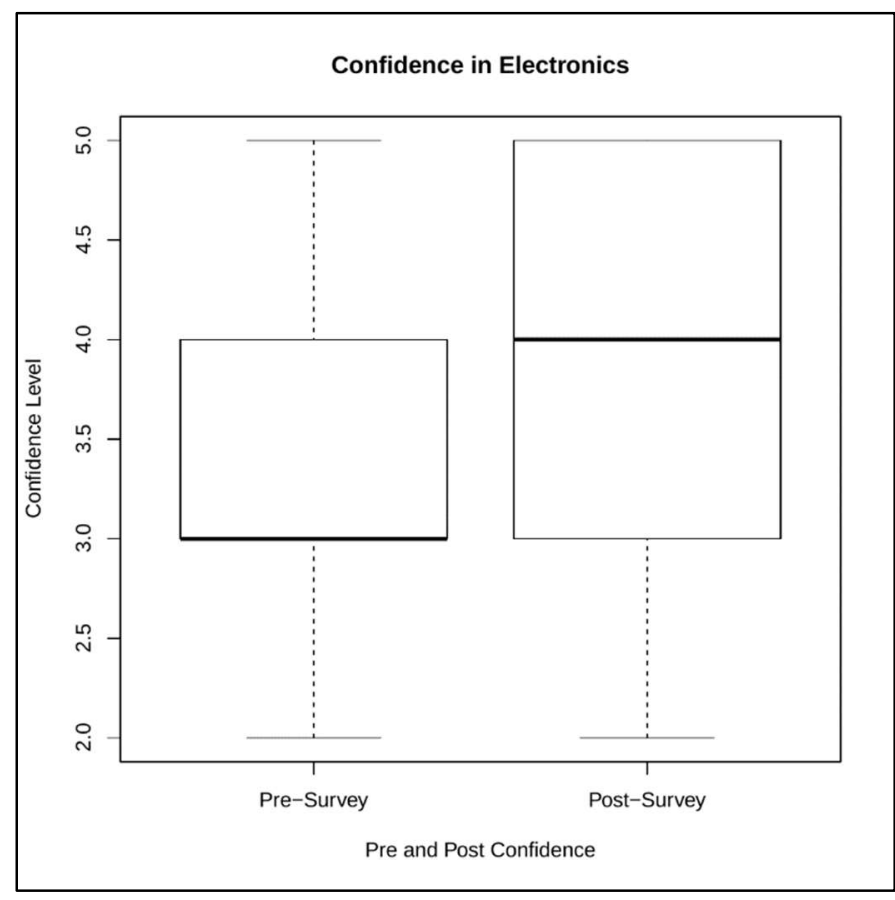

Figure 5. Differences of Pre and Post Survey Results for Confidence in Electronics

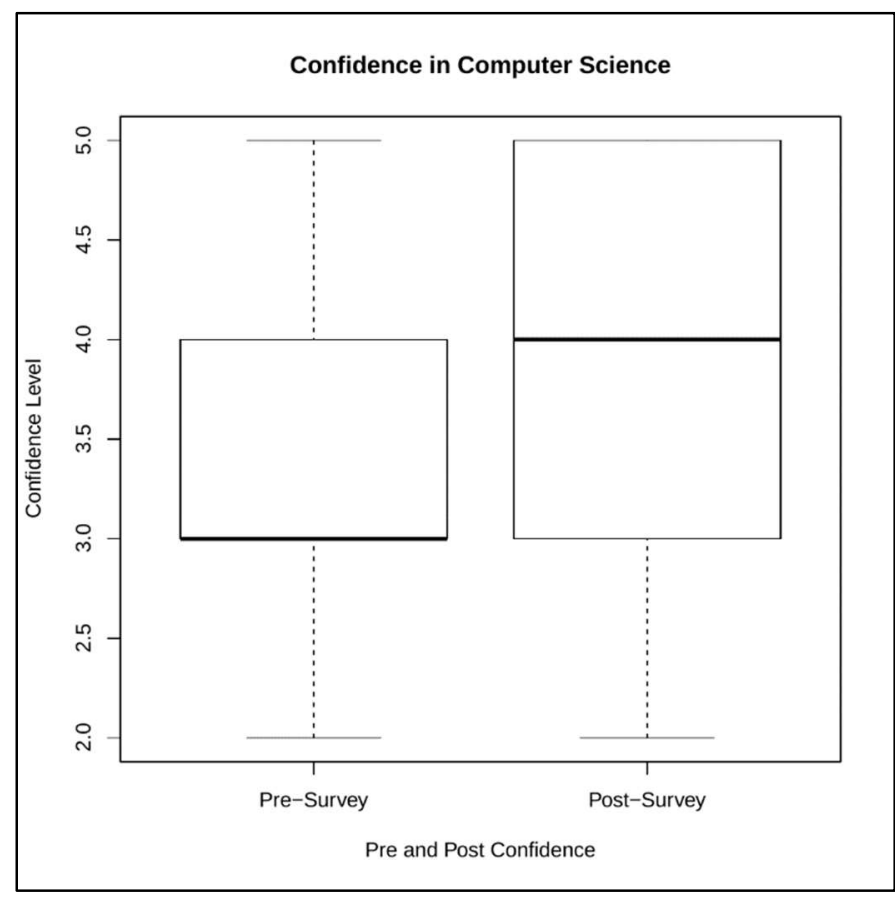

Figure 6. Differences of Pre and Post Survey Results for Confidence in Computer Science 
This research also finds that there was a significant change in the answers provided to quiz question 3 (shown in Figure 3 above) before and after the intervention ( $p$-value $=0.0011$ ). This question showed a circuit schematic containing a resistor in series with an LED in an incorrect orientation. The question then asked if the LED was in the correct orientation. The frequency of incorrect answers increased by 14 points from pre-quiz to post-quiz, even though students were exposed to the correct schematic with the correct orientation during the ActivityBot $360^{\circ}$ tutorials.

A Kruskal-Wallis Test was used to determine if demographic groups differed significantly in how their answers change for the 3 out of 4 confidence topics and quiz question 3. This test takes in survey and demographic data for a population and returns a specific $p$-value. P-values that are less than the 0.05 significance level represents that the demographic groups being compared differ significantly. Students with different experience levels in electronics differ in the confidence with robotics before and after the intervention (see Fig. 7), and students who did not choose to take the STE(A)M elective differed in their confidence in electronics compared to students who did choose to take the elective (see Fig. 8). There are no differences among any other demographic groups.

Shown in Figure 7, students who did not have experience with electronics (33 out of 57 students) had a greater increase in confidence in robotics than the students who already had experience with electronics ( 24 out of 57 students) ( $p$-value $=0.0423$ ). It should be noted that initially only $33.33 \%$ of the students who did not have experience with electronics stated positive confidence (slightly and very confident), and this grew to $63.64 \%$ of the students after the robotics intervention. This is different than the $70.83 \%$ of the students who already had experience with electronics initially stating that they had positive confidence, and their positive confidence grew to $79.17 \%$ after the intervention. Students without experience had lower confidence levels when compared to experienced students, but the students without experience had a significantly higher gain in confidence. 


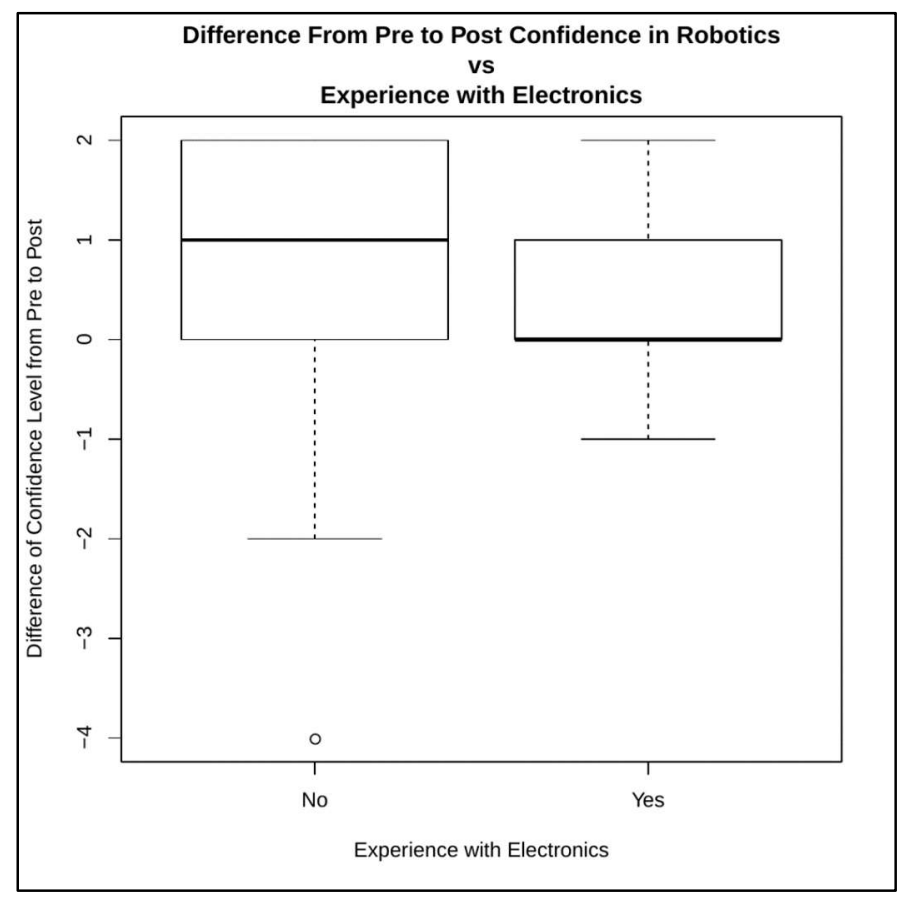

Figure 7. Experience with Electronics and Change in Confidence in Robotics

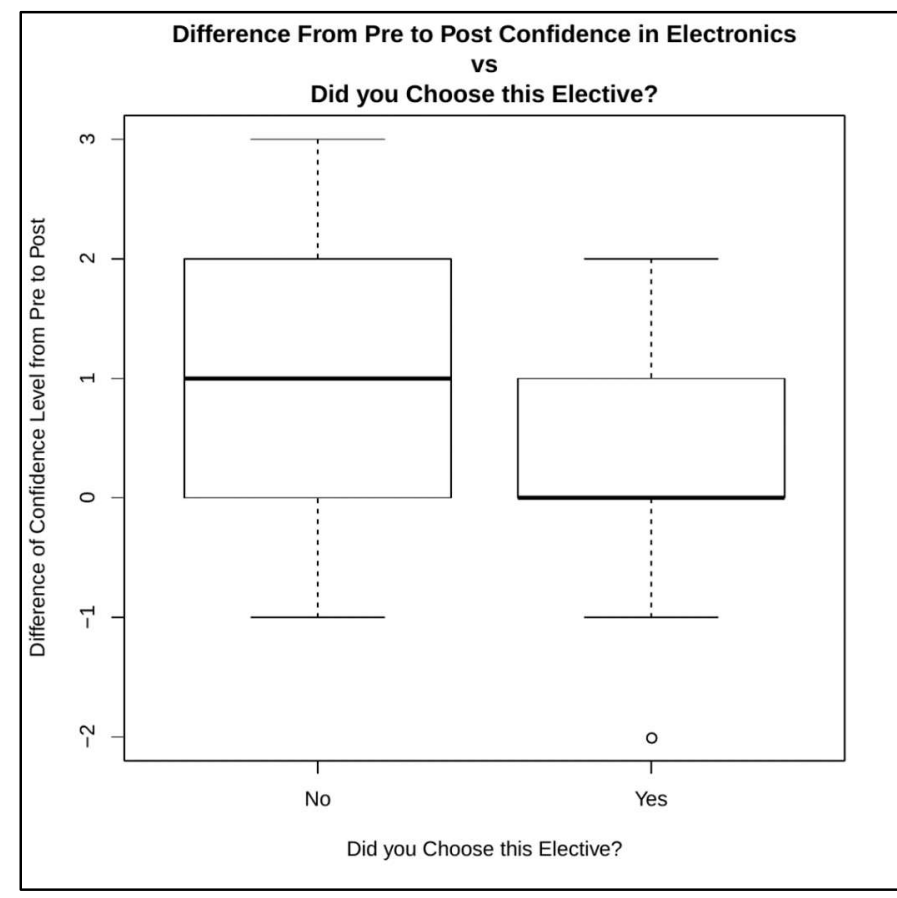

Figure 8. Choosing to Take the STE(A)M Elective and Change in Confidence in Electronics 
Shown in Figure 8, students who did not choose to take this elective (20 out of 57 students) had a greater increase in confidence in electronics when compared to the group of students who did choose to take the elective ( 37 out of 57 students) (p-value $=0.0426$ ). It should be noted that only $30.00 \%$ of the students who did not choose this elective had a positive confidence initially, and this positive confidence grew to $65.00 \%$ of the students after the intervention. In comparison, $56.76 \%$ of the students who did choose to take the elective initially had a positive confidence in electronics, and this percentage rose to $75.68 \%$.

\section{Conclusion}

While there were no significant correlations between exposure to the ActivityBot $360^{\circ}$ and interest, enjoyment, and motivation to pursue a degree/career in STEM, there was a significant increase in confidence in robotics, electronics, and computer science. This study shows that the exposure to the ActivityBot $360^{\circ}$, its tutorial, and instruction from a teacher significantly increased the general confidence of $8^{\text {th }}$ grade middle school students in a CTE STE(A)M elective in these three STEM areas. It also shows that familiarizing students with parts and schematics can possibly create confusion, but further research needs to be conducted to confirm why less students got Question 3 correct. In addition, the study shows that students who had no experience with building or designing electronics had, on average, a greater increase in confidence in robotics than students who already had experience, and students who did not choose this elective had, on average, a greater increase in confidence in electronics than students who did choose to take this elective.

While this research study did not compare a control and experimental group to determine learning outcomes like Zygouris et al., who found that the group of students who used Lego Mindstorms to learn geometry performed better on a questioner when compared to the group of students who received a normal lesson [9], this research does compare students' interests, motivation, confidence, enjoyment, and knowledge before and after the intervention. Zygouris et al. focused on using robotics to teach students specific math concepts that were already being taught without robotics, while our research focused on the impacts of exposing students to 
robotics and the general knowledge they gain about electronics and programming as a result of the exposure.

This research study is more similar to Knop et al. because the "Neu-pulator platform" presented more electronics than the Lego Mindstorms [8]. The robot used an Arduino platform, which is like the Parallax Propeller Board with various sensors and motors. Knop et al. found an increase in students' confidence towards STEM topics [8], which was further supported by this research. However, Knop et al. find that students' interest towards robotics increases, which this research does not support, but this difference in findings might be due to the difference in population. The students in the Knop et al. were in a summer program; whereas, the participants in this study were from a dual-language immersion middle school.

Insights

Students who chose the elective typically stated that they had previous exposure to STEM topics. This statistic combined with the significant increase in confidence for inexperienced students in electronics shows the need for exposing inexperienced students to robotics and other STEM topics. Even if a student does not have any interest in STEM, it is possible that positive confidence might make one believe that they could be someone who can pursue STEM. Without exposure to the technology and engineering fields in STEM, students may never gain confidence in these fields and possibly keep a fixed mindset about the fields or be discouraged from pursuing the ever-growing fields in STEM.

For Question 3, the tutorial may have caused confusion in which way an LED should be orientated, but since the majority of the students did not have prior experience with electronics, it is probable that students were answering randomly. It should be noted that the teacher for this classroom is not trained in electronics, robotics, or computer science, but she is trained in mathematics. This means that even if a student did understand the physical LED orientation, they may not have understood the schematic which was shown in the tutorial and were unable to be given further information about the circuit schematic. 
Recommendations for Educators

Even though this research shows that exposure to the ActivityBot $360^{\circ}$ can increase students' confidence, it should be noted that for students to truly learn from this device there needs to be an instructor present who is trained in robotics, electronics, and/or computer science. Students can only learn so much from active learning on their own. With better trained teachers and an improved curriculum, students can more effectively learn to not only make the robot work but learn why it works as well.

While students' motivations to pursue STEM fields did not increase as a result of the intervention, it can be suggested that students already have a strong opinion in what fields they would like to pursue by the 8th grade, which means even earlier exposure could make a stronger impact in shaping their attitudes. However, the significant increase in confidence among those students without experience to technology and engineering fields suggests that exposure at any time is good and those who might not self-select into a STEM elective might have the most to gain from it. In addition, making it mandatory for all students to take a STEM CTE course could help improve equity for students who lack the resources to STEM exposure. While paired and collaborative learning has shown to improve the educational experience, providing a 1 to 1 student to device (computer, robot, etc.) ratio can also be a way to make sure all students in this course are equally exposed to the technology and engineering topics.

\section{References}

[1] P. Plaza, E. Sancristobal, G. Carro, M. Blazquez, F. García-Loro, S. Martin, C. Perez, and M. Castro, "Arduino as an educational tool to introduce robotics," in 2018 IEEE International Conference on Teaching, Assessment, and Learning for Engineering (TALE), Dec 2018, pp. 1-8.

[2] Z. Pei and Y. Nie, "Educational robots: Classification, characteristics, application areas and problems," in 2018 Seventh International Conference of Educational Innovation through Technology (EITT), Dec 2018, pp. 57-62.

[3] M. Petre and B. Price, "Using robotics to motivate 'back door' learning," Education and Information Technologies, vol. 9, pp. 147-158, 062004.

[4] V. Chaudhary, V. Agrawal, P. Sureka, and A. Sureka, "An experience report on teaching programming and computational thinking to elementary level children using lego robotics 
education kit," in 2016 IEEE Eighth International Conference on Technology for Education (T4E), Dec 2016, pp. 38-41.

[5] Oregon Department of Education, "High school graduation and college and career readiness act," Oct 2017. [Online]. Available: https://www.oregon.gov/ode/students-andfamily/GraduationImprovement/Documents/High\%20School\%20Graduation\%20And\%2 0College\%20and\%20Career\%20Readiness\%20Act.pdf. [Accessed Oct, 2019]

[6] Oregon Department of Education, "High school success frequently asked questions," Sept 2019. [Online]. Available: https://www.oregon.gov/ode/students-andfamily/GraduationImprovement/Documents/HSS\%20FAQ.pdf. [Accessed Oct, 2019]

[7] Parallax, "Blocklyprop robotics with the activitybot 360," pp. 1-3, Aug 2018. [Online]. Available: https://learn.parallax.com/tutorials/robot/activitybot/blocklyprop-roboticsactivitybot-360\%C2\%B0. [Accessed Aug, 2018]

[8] L. Knop, S. Ziaeefard, G. A. Ribeiro, B. R. Page, E. Ficanha, M. H. Miller, M. Rastgaar, and N. Mahmoudian, "A human-interactive robotic program for middle school stem education," in 2017 IEEE Frontiers in Education Conference (FIE), Oct 2017, pp. 1-7.

[9] N. C. Zygouris, A. Striftou, A. N. Dadaliaris, G. I. Stamoulis, A. C. Xenakis, and D. Vavougios, "The use of lego mindstorms in elementary schools," in 2017 IEEE Global Engineering Education Conference (EDUCON), April 2017, pp. 514-516. 